\title{
Wireless Energy and Information Transmission in FSO and RF-FSO Links
}

\author{
Behrooz Makki, Tommy Svensson, Koen Buisman, Joaquin Perez and Mohamed-Slim Alouini, Fellow, IEEE
}

\begin{abstract}
We propose and analyze a wireless energy and information transmission scheme in free-space optical (FSO) links. The results are presented for both quasi-static and fast-fading conditions. We derive closed-form expressions for throughput, outage probability and optimal power allocation optimizing the system throughput/outage probability. Finally, we complement the FSO link with an additional radio frequency (RF) link to create a hybrid RF-FSO system and reduce the system outage probability. The results show that joint implementation of the RF and FSO links leads to considerable performance improvement, compared to the cases with only FSO-based communication.
\end{abstract}

\section{INTRODUCTION}

Future wireless networks are expected to be densified with a large number of simpler and cheaper nodes that will be deployed for different applications. Some nodes may be powered by batteries with limited life-time, which are periodically replaced by the operator. However, self-sustaining nodes which harvest their energy from the surrounding environment have received considerable attention recently. Particularly, in various sensor networks, the power consumption of the nodes is in the order of $\mu \mathrm{W}$, which corresponds to the range where energy harvesting from the environment is possible. Also, these nodes are mostly used for bursty communications and, as a result, the required energy can be effectively harvested within successive packet transmissions. Here, particular attention is paid to wireless energy and data transfer setups where the transmitter charges the receiver before data transfer [1], [2].

From another perspective, free-space optical (FSO) systems are powerful tools for transferring data over short distances [3]-[5]. FSO systems provide fiber-like data rates through the atmosphere using lasers or light emitting diodes (LEDs) with considerably lower cost, compared to fiber links, and without extensive installation needs such as digging. For these reasons, and also because of the line-of-sight (LOS) propagation nature of the FSO link with narrow beams, it is an attractive candidate for wireless energy and information transmission setups, as recently considered in industry, e.g., [6]. Also, the combination of FSO and radio frequency (RF) links in a hybrid RF-FSO setup is considered as a strong candidate to improve the network outage probability because each link can compensate for the other link when it is down [4], [5].

In this letter, we develop and analyze a wireless energy and data transmission scheme over FSO links. With a limit

Behrooz Makki, Tommy Svensson and Koen Buisman are with Chalmers University of Technology, Gothenburg, Sweden, Email: \{behrooz.makki, tommy.svensson, buisman $\} @$ chalmers.se. Joaquin Perez is with Universitat Politecnica de Valencia, Valencia, Spain, Email: joapeso@upv.es. MohamedSlim Alouini is with the King Abdullah University of Science and Technology, Thuwal, Saudi Arabia, Email: slim.alouini@kaust.edu.sa

978-1-4799-5863-4/14/\$31.00 (C)2014 IEEE on the total energy and information transmission delay, the design problem is cast in the form of optimizing the outage probability/throughput subject to a total energy consumption constraint. We present the results for both quasi-static and fastfading conditions. Also, we derive closed-form expressions for the system throughput, the outage probability and the optimal power allocation, in terms of throughput/outage probability. Finally, we investigate the possibility of improving the system performance via RF-FSO based communication.

Our results indicate that, compared to FSO-based communication, the joint RF-FSO based communication reduces the energy-limited outage probability considerably. On the other hand, for a broad range of parameter settings, the optimal power allocation between energy and information signals has marginal effect on the outage probability/throughput. Finally, while the presence of a battery improves the outage probability, its effect is negligible for moderate/large code rates.

\section{System MOdEL}

Consider an FSO link utilizing coherent model during data transmission [3]. A packet transmission phase is defined as the transmission of a codeword and its required energy. We assume the transmitter to be equipped with a permanent power supply. However, the receiver has no power supply, and is charged by the transmitter at the beginning of each packet transmission. In this way, a packet of length $2 L$ channel uses (cu) is divided into two slots of length $L \mathrm{cu}$. In the first slot, the transmitter sends the energy signal to the receiver. Then, if the receiver's minimum required energy is supplied in Slot 1 , the remaining $L \mathrm{cu}$ are utilized for information communication in the second slot. The energy and information signals are transferred to the receiver in different frequency bands, motivated by different properties of energy harvesting and information reception circuits [7].

We consider a bursty communications model [2] where there is a long random idle period between two successive packet transmissions. Also, as an appropriate model for the FSO channel, the results are given for the Gamma-Gamma distribution of the FSO link where the energy and data transmission channel gains follow the probability density function $(\mathrm{PDF})^{1}$

$$
f_{G}(x)=\frac{2(a b)^{\frac{a+b}{2}}}{\Gamma(a) \Gamma(b)} x^{\frac{a+b}{2}-1} K_{a-b}(2 \sqrt{a b x}),
$$

with $K_{n}(\cdot)$ denoting the modified Bessel function of the second kind of order $n$ and $\Gamma(\cdot)$ being the Gamma function. Also, $a$ and $b$ are the distribution shaping parameters.

${ }^{1}$ For simplicity of notations, we consider the same channel models for the energy and information transmission links. However, it is straightforward to extend the results to the cases with different channel models. 


\section{ANALYTICAL RESULTS}

Let us define $g_{\mathrm{E}}$ and $g_{\mathrm{I}}$ as the channel gains during the energy and information transmission phases, respectively. In this way, the accumulated energy at the end of the energy transfer phase is given by $E_{\text {received }}=\delta P_{\mathrm{E}} g_{\mathrm{E}} L$ where $P_{\mathrm{E}}$ denotes the power of the energy transfer signal and $\delta \leq 1$ is an efficiency parameter depending on the energy absorption properties of the energy harvesting circuit. The receiver is successfully charged at the end of Slot 1 , if $E_{\text {received }} \geq E_{\text {required }}$, i.e., $g_{\mathrm{E}} \geq \frac{\theta}{\delta P_{\mathrm{E}}}$, where $E_{\text {required }}$ is the minimum energy required by the receiver for information processing and we define a normalized energy requirement factor $\theta \doteq \frac{E_{\text {required }}}{L}$ (the energy cost of channel estimation and sending one acknowledgement feedback bit at the end of Slot 1 is included in $\left.E_{\text {required }}\right)$. Otherwise, circuit outage occurs if $g_{\mathrm{E}}<\frac{\theta}{\delta P_{\mathrm{E}}}$.

Given that the receiver has successfully accumulated the required energy, the data transmission starts in the second time slot and the message is correctly decoded by the receiver if $\log \left(1+P_{\mathrm{I}} g_{\mathrm{I}}\right) \geq R$. Here, $P_{\mathrm{I}}$ represents the power of the information transfer signal. Also, $R=\frac{K}{L}$ in nats-per-channeluse (npcu) denotes the code rate with $K$ being the number of nats per codeword. In this way, the system successful message decoding probability and the throughput are given by

$$
\begin{aligned}
& \operatorname{Pr}(\text { Success })=\operatorname{Pr}\left(g_{\mathrm{E}} \geq \frac{\theta}{\delta P_{\mathrm{E}}}\right) \operatorname{Pr}\left(\log \left(1+P_{\mathrm{I}} g_{\mathrm{I}}\right) \geq R\right) \\
& =\left(1-F_{G}\left(\frac{\theta}{\delta P_{\mathrm{E}}}\right)\right)\left(1-F_{G}\left(\frac{e^{R}-1}{P_{\mathrm{I}}}\right)\right),
\end{aligned}
$$

and

$$
\begin{aligned}
\eta & =\frac{R}{2} \operatorname{Pr}(\text { Success }) \\
& =\frac{R}{2}\left(1-F_{G}\left(\frac{\theta}{\delta P_{\mathrm{E}}}\right)\right)\left(1-F_{G}\left(\frac{e^{R}-1}{P_{\mathrm{I}}}\right)\right),
\end{aligned}
$$

respectively, where

$$
F_{G}(x)=\frac{1}{\Gamma(a) \Gamma(b)} \mathcal{G}_{1,3}^{2,1}\left(\left.a b x\right|_{a, b, 0} ^{1}\right),
$$

is the cumulative distribution function (CDF) of the GammaGamma distribution with $\mathcal{G}(\cdot)$ denoting the Meijer G-function. Also, the outage probability is $\operatorname{Pr}$ (Outage) $=1-\operatorname{Pr}$ (Success). Finally, the total energy consumed by the transmitter for energy transfer in Slot 1 and possibly data transfer in Slot 2 , normalized by the codeword length, is obtained as

$$
\Xi=P_{\mathrm{E}}+P_{\mathrm{I}}\left(1-F_{G}\left(\frac{\theta}{\delta P_{\mathrm{E}}}\right)\right) \text {. }
$$

In this way, the throughput and the outage probability are monotonic functions of the successful message decoding probability $\operatorname{Pr}$ (Success). Thus, with a sum normalized energy constraint $\Xi=\xi$, the energy-limited outage probability/throughput optimization problem can be represented as

$$
\left\{\begin{array}{l}
\max _{P_{\mathrm{E}}, P_{\mathrm{I}}}\left(1-F_{G}\left(\frac{\theta}{\delta P_{\mathrm{E}}}\right)\right)\left(1-F_{G}\left(\frac{e^{R}-1}{P_{\mathrm{I}}}\right)\right) \\
\text { subject to } P_{\mathrm{E}}+P_{\mathrm{I}}\left(1-F_{G}\left(\frac{\theta}{\xi P_{\mathrm{E}}}\right)\right)=\xi,
\end{array}\right.
$$

with $P_{\mathrm{E}}$ and $P_{\mathrm{I}}$ being the optimization variables. In general, (6) does not have a closed-form solution, due to the complex CDF (4). For this reason, Theorem 1 presents a high signalto-noise ratio (SNR) approximate solution for (6) as follows.
Theorem 1. As moderate/high SNRs, the optimal power allocation, in terms of energy-limited outage probability/throughput, tends towards

$$
\left\{\begin{array}{c}
P_{\mathrm{E}}=\frac{\xi\left(\frac{\theta}{\delta\left(e^{R}-1\right)}\right)^{\frac{\tau}{1+\tau}}}{1+\left(\frac{\theta}{\delta\left(e^{R}-1\right)}\right)^{\frac{\tau}{1+\tau}}}, \tau=\min \{a, b\} . \\
P_{\mathrm{I}}=\frac{\xi}{1+\left(\frac{\theta}{\delta\left(e^{R}-1\right)}\right)^{\frac{\tau}{1+\tau}}} .
\end{array}\right.
$$

Proof. At high SNRs, one can use [3, Lemmas 1-2] to approximate the PDF (1) by

$$
\begin{aligned}
& f_{G}(x) \sim\left(\frac{\Gamma(v-\tau)(v \tau)^{\tau}}{\Gamma(v)}\right) \frac{x^{\tau-1}}{\Gamma(\tau)}, \\
& \tau=\min \{a, b\}, v=\max \{a, b\} .
\end{aligned}
$$

Also, the normalized energy constraint of (6) is simplified to $P_{\mathrm{E}}+P_{\mathrm{I}}=\xi$, because the receiver is successfully charged with high probability as the SNR increases. In this way, defining the constant $c=\frac{\Gamma(v-\tau)(v \tau)^{\tau}}{\tau \Gamma(v) \Gamma(\tau)},(6)$ is approximated by

$$
\begin{aligned}
& \left\{\begin{array}{l}
\max _{P_{\mathrm{E}}, P_{\mathrm{I}}}\left(1-c\left(\frac{\theta}{\delta P_{\mathrm{E}}}\right)^{\tau}\right)\left(1-c\left(\frac{e^{R}-1}{P_{\mathrm{I}}}\right)^{\tau}\right) \\
\text { subject to } P_{\mathrm{E}}+P_{\mathrm{I}}=\xi,
\end{array}\right. \\
& \stackrel{(a)}{\simeq}\left\{\begin{array}{c}
\min _{P_{\mathrm{E}}, P_{\mathrm{I}}}\left(\frac{\theta}{\delta P_{\mathrm{E}}}\right)^{\tau}+\left(\frac{e^{R}-1}{P_{\mathrm{I}}}\right)^{\tau} \\
\text { subject to } P_{\mathrm{E}}+P_{\mathrm{I}}=\xi,
\end{array}\right.
\end{aligned}
$$

which leads to (7). In (9), (a) comes from removing the smallest terms at high SNRs. Then, (7) is obtained by replacing $P_{\mathrm{I}}=\xi-P_{\mathrm{E}}$ into the objective function of (9) and setting the derivative of the objective function equal to zero.

As shown in Section IV, while (7) is found by the high-SNR approximation, it is tight for a broad range of SNRs. Also, (7) follows intuition where with high energy requirements, low energy absorption efficiency or low data rates, i.e., high values of $\theta$, low values of $\delta$ or low $R$ 's, respectively, most of the available energy should be allocated to the energy transfer signal. Also, (7) indicates that the optimal power of the energy transfer signal scales with the normalized total energy $\xi$ linearly. On the other hand, for large values of $R$, the optimal power of the energy transfer signal decreases with the code rate $R$ almost exponentially.

Finally, note that because the receiver receives the energy and information signals via different circuits, one can use simultaneous transmission schemes where the energy and information signals are sent in the same time slot. However, for the network simplicity and also because of the total energy constraint, we concentrate on the sequential transmission scheme where the energy can be saved by sending the information signal only if enough energy has been harvested.

\section{A. Performance Analysis in Fast-fading Conditions}

Up to now, the results were presented for cases with long coherence times where the channel gains remain constant during the energy and data transmission periods. However, it is interesting to analyze the system performance in fast-fading conditions as well as frequency hopping-based systems where multiple channel realizations are experienced in each time slot. 
Considering $N$ different channel realizations during the message transmission period, the message outage probability is given by

$$
\operatorname{Pr}(\text { Message outage })=\operatorname{Pr}\left(\frac{1}{N} \sum_{i=1}^{N} \log \left(1+P_{\mathrm{I}} g_{\mathrm{I}}^{i}\right) \leq R\right),
$$

where $g_{\mathrm{I}}^{i}$,s are different channel realizations during data transmission. Using Minkowski inequality [8, Theorem 7.8.8]

$$
\left(1+\left(\prod_{i=1}^{n} x_{i}\right)^{\frac{1}{n}}\right)^{n} \leq \prod_{i=1}^{n}\left(1+x_{i}\right)
$$

we have

$$
\begin{aligned}
\operatorname{Pr}(\text { Message outage }) & \leq \operatorname{Pr}\left(1+P_{\mathrm{I}}\left(\prod_{i=1}^{N} g_{\mathrm{I}}^{i}\right)^{\frac{1}{N}} \leq e^{R}\right) \\
& =F_{\mathcal{Q}}\left(\left(\frac{e^{R}-1}{P_{\mathrm{I}}}\right)^{N}\right)
\end{aligned}
$$

where the $\mathrm{CDF}$ of $\mathcal{Q}=\prod_{i=1}^{N} g_{\mathrm{I}}^{i}$ is given by [3, Lemma 3]

$$
F_{\mathcal{Q}}(x)=\frac{1}{\Gamma^{N}(a) \Gamma^{N}(b)} \mathcal{G}_{1,2 N+1}^{2 N, 1}(\left.(a b)^{N} x\right|_{\underbrace{}_{N \text { times }}} ^{1, \ldots, a}, \underbrace{b, \ldots, b, 0}_{N \text { times }}) \text {. }
$$

Also, the circuit outage probability is found as

$\operatorname{Pr}($ Circuit outage $)=\operatorname{Pr}\left(\frac{1}{N} \sum_{i=1}^{N} g_{\mathrm{E}}^{i} \leq \frac{\theta}{\delta P_{\mathrm{E}}}\right)=F_{\mathcal{B}}\left(\frac{N \theta}{\delta P_{\mathrm{E}}}\right)$

where $\mathcal{B} \doteq \sum_{i=1}^{N} G_{i}$ a random variable following the PDF [3, Lemma 1]

$$
\begin{aligned}
& f_{\mathcal{B}}(x)=\frac{2\left(\frac{\sigma_{N} S N}{N} x\right)^{\frac{\sigma_{N}+\varsigma_{N}}{2}} K_{\sigma_{N}-\varsigma_{N}}\left(2 \sqrt{\frac{\sigma_{N S N}}{N} x}\right)}{\Gamma\left(\sigma_{N}\right) \Gamma\left(\varsigma_{N}\right) x}, \\
& \sigma_{N}=N v+\varrho_{N}, \\
& \varsigma_{N}=N \tau, \tau=\min \{a, b\}, v=\max \{a, b\},
\end{aligned}
$$

with $\varrho_{m N}$ being an appropriately chosen adjustment parameter $^{2}$. Using (12)-(14), one can bound the successful message decoding probability, the throughput and the total consumed energy, and analyze the performance of the proposed setup in fast-fading and frequency hopping-based channels.

\section{B. Performance Improvement via RF-FSO Communication}

Under severe weather conditions, e.g., strong atmospheric turbulence, the FSO performance suffers and goes down. An efficient method for increasing the system availability is to complement the FSO link with an additional RF link, creating a hybrid RF-FSO communication system. Here, along with joint transmission, e.g., [4], particular attention is paid to serial RF-FSO setups where the RF link acts as a backup when the FSO link is down, e.g., [5].

We consider the scenario where, given that the FSObased message transmission fails in the second time slot,

\footnotetext{
${ }^{2}$ Equation (15) gives an approximation of the sum of IID Gamma-Gamma variables. However, as shown in, e.g., [3], the approximation is extremely tight for all ranges of SNRs. Therefore, we consider it as an equality.
}

an additional time slot of length $L \mathrm{cu}$ is used to retransmit the information through an RF-based link ${ }^{3}$. In this case, the successful message decoding probability (2) is rephrased as

$$
\begin{aligned}
& \operatorname{Pr}(\text { Success })=\operatorname{Pr}\left(g_{\mathrm{E}} \geq \frac{\theta}{\delta P_{\mathrm{E}}}\right)\left(\operatorname{Pr}\left(\log \left(1+P_{\mathrm{I}} g_{\mathrm{I}}\right) \geq R\right)\right. \\
& \left.+\operatorname{Pr}\left(\log \left(1+P_{\mathrm{I}} g_{\mathrm{I}}\right)<R\right) \operatorname{Pr}\left(\log \left(1+\tilde{P}_{\mathrm{I}} \tilde{g}_{\mathrm{I}}\right) \geq R\right)\right) \\
& =\left(1-F_{G}\left(\frac{\theta}{\delta P_{\mathrm{E}}}\right)\right)\left(1-F_{G}\left(\frac{e^{R}-1}{P_{\mathrm{I}}}\right)\right. \\
& \left.+F_{G}\left(\frac{e^{R}-1}{P_{\mathrm{I}}}\right)\left(1-F_{\tilde{G}}\left(\frac{e^{R}-1}{\tilde{P}_{\mathrm{I}}}\right)\right)\right),
\end{aligned}
$$

if the channels follow quasi-static fading conditions (using the same procedure as in Section III.A, one can extend the results to the cases with fast-fading conditions). In (16), $\tilde{P}_{\mathrm{I}}$ and $\tilde{g}_{\mathrm{I}}$ denote the power of the RF-based signal and the channel gain of the RF-based link, respectively, and considering Rician fading, which is an appropriate model for LOS conditions, the $\mathrm{RF}$ channel gain follows the $\mathrm{CDF}$

$$
F_{\tilde{G}}(x)=1-\mathcal{Q}\left(\frac{\mu}{\omega}, \frac{\sqrt{x}}{\omega}\right) .
$$

Here, $\mathcal{Q}(\cdot, \cdot)$ denotes the Marcum Q function and $\mu$ and $\omega$ are the long-term channel parameters of the Rician channel. Then, the throughput (3) and the total consumed energy (5) are respectively rephrased as

$$
\begin{aligned}
\eta & =\frac{K \operatorname{Pr}(\text { Success })}{\Delta}=\frac{R \operatorname{Pr}(\text { Success })}{1+\left(1-F_{G}\left(\frac{\theta}{\delta P_{\mathrm{E}}}\right)\right)\left(1+F_{G}\left(\frac{e^{R}-1}{P_{\mathrm{I}}}\right)\right)} \\
\Delta & \doteq L+L \operatorname{Pr}\left(g_{\mathrm{E}} \geq \frac{\theta}{\delta P_{\mathrm{E}}}\right)\left(\operatorname{Pr}\left(\log \left(1+P_{\mathrm{I}} g_{\mathrm{I}}\right) \geq R\right)\right. \\
& \left.+2 \operatorname{Pr}\left(\log \left(1+P_{\mathrm{I}} g_{\mathrm{I}}\right)<R\right)\right)
\end{aligned}
$$

and

$$
\Xi=P_{\mathrm{E}}+\left(1-F_{G}\left(\frac{\theta}{\xi P_{\mathrm{E}}}\right)\right)\left(P_{\mathrm{I}}+\tilde{P}_{\mathrm{I}} F_{G}\left(\frac{e^{R}-1}{P_{\mathrm{I}}}\right)\right) .
$$

Also, from (16)-(19), one can determine the optimal power terms optimizing the outage probability/throughput with a total energy constraint.

Note that setting $\tilde{P}_{\mathrm{I}}=0$ in (16) and (19), which is not necessarily optimal for the RF-FSO based setup, the outage probability and the total energy of the RF-FSO based setup are simplified to those with only FSO-based communication. Thus, in the optimal case and with the same $\theta$, the RF-FSO setup outperforms the FSO-based system in terms of energylimited outage probability. However, in practice, adding the RF link may increase the normalized required energy $\theta$ (see Figs. 1a-1b). Also, as opposed to outage probability, depending on the parameter settings, the use of the RF link may or may not improve the throughput. This is because, while the presence of the RF-based link increases the successful message decoding probability, it increases the total number of channel uses required for a packet transmission.

${ }^{3}$ Following the same procedure as in [2], one can extend the results to the cases where the RF link is used for both energy and information transfer. 

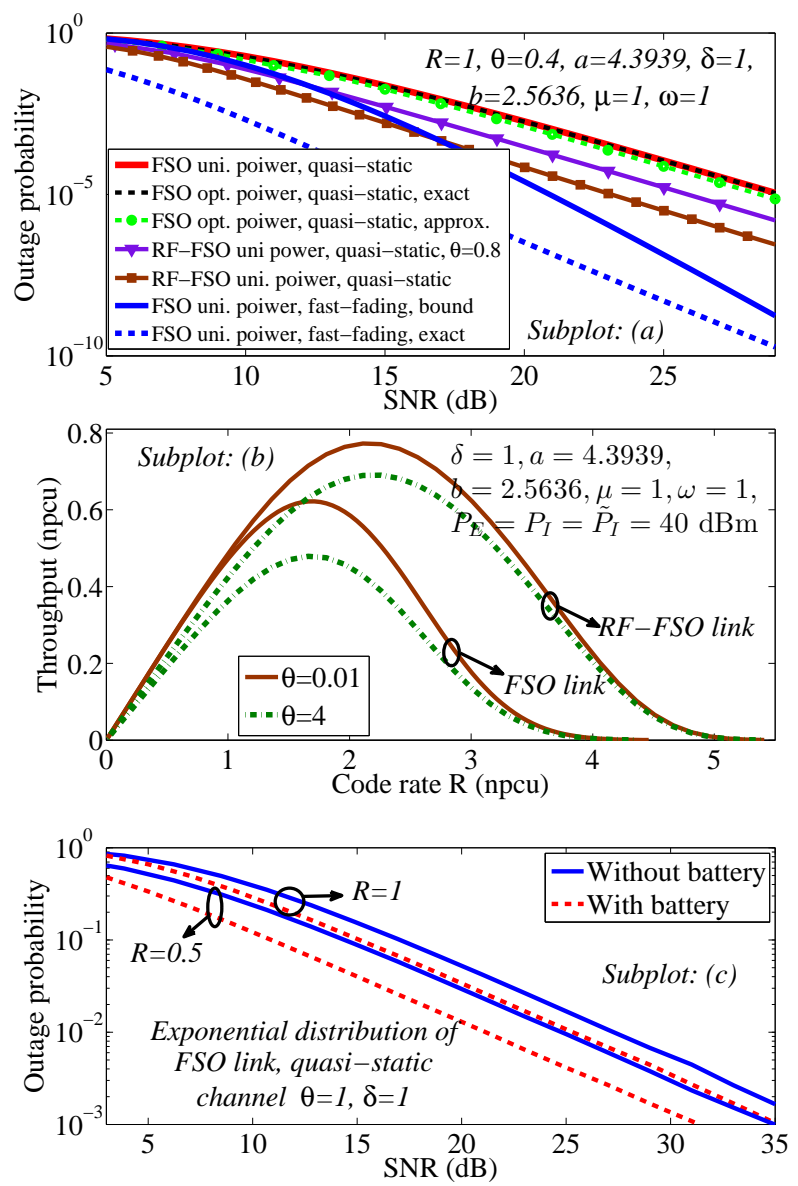

Figure 1. (a): Outage probability for different communication setups. (b): Throughput vs the code rate $R$. (c): On the effect of the battery on the outage probability of the FSO link, exponential PDF of the FSO link.

\section{Simulation Results And Conclusions}

We set $a=4.3939$, and $b=2.5636$, which correspond to Rytov variance 1 of the FSO link [3], and set the parameters of Rician RF PDF to $\omega=1, \nu=1$. Also, we consider $\delta=1$, $\theta=1$, unless otherwise stated. In Figs. 1a and 1c, we consider an average normalized energy constraint $\Xi=\xi$ and, because the variance of the noise at the information receiver is 1 , we refer to $\xi$ (in $\mathrm{dB}, 10 \log _{10} \xi$ ) as SNR. In Fig. 1b, however, we present the results for the cases with peak power constraints.

Setting $R=1 \mathrm{npcu}$ and $N=2$ in fast-fading conditions, Fig. 1a shows the outage probability for different communication setups, and verifies the tightness of the proposed approximations/bounds. Here, uniform power allocation stands for the case with $P_{\mathrm{E}}=P_{\mathrm{I}}=\tilde{P}_{\mathrm{I}}$. Then, Fig. $1 \mathrm{~b}$ shows the throughput for different code rates $R$ and normalized energy requirements $\theta$. Here, the results are presented for peak powers $P_{\mathrm{E}}=P_{\mathrm{I}}=\tilde{P}_{\mathrm{I}}=40 \mathrm{dBm}$. Also, Figs. 1a-b compare the performance of the FSO and RF-FSO based schemes, in terms of outage probability and throughput, respectively.

Throughout the paper, we considered the Gamma-Gamma PDF for the FSO link. However, we can redo the analysis for other, e.g., the exponential PDFs $f_{G}(x)=e^{-x}$. Also, motivated by the bursty communication model and the simplicity of the energy harvesting nodes, we ignored the effect of longterm energy storage batteries where the remaining energy of each packet transmission is stored in a battery. In Fig. 1c, we verify the system performance for the cases with exponential PDF of the FSO link and study the effect of the long-term batteries on the outage probability. According to the figures, the following conclusions can be drawn:

1) The approximation scheme of Theorem 1 is tight for a broad range of parameter settings/SNRs. Also, (12)-(15) properly upper-bound the outage probability in fast-fading conditions (Fig. 1a). Thus, the results of Section III can be well utilized for the analytical performance evaluation of wireless energy and information transfer systems.

2) For a broad range of SNRs/parameter settings, adaptive power allocation between the energy and data transmission signals has marginal effect on the throughput/outage probability (Fig. 1a). Thus, considering the complexity of adaptive power allocation, uniform power allocation is preferable in practice.

3) Compared to quasi-static model, the outage probability of the FSO link is reduced considerably in fast-fading conditions. This is intuitive because more time diversity is exploited when the channel changes during energy/data transmission (Fig. 1a).

4) For low $R$ 's, the throughput increases with the rate (almost) linearly, because with high probability the data is correctly decoded. On the other hand, the outage probability increases and the throughput goes to zero for large values of $R$ (Fig. 1b). Also, as expected, the throughput decreases with the energy requirement $\theta$.

5) For a broad range of energy requirements $\theta$, the RF-FSO link leads to substantially less outage probability, compared to the cases with only FSO-based communication. Intuitively, this is because the RF link compensates the effect of the FSO link, if it experiences poor channel conditions (Fig. 1a). Also, with the parameter setting of Fig. 1b, the RF-FSO system outperforms the FSO-based setup, in terms of throughput.

6) Finally, as seen in Fig. 1c, the same approach as in the cases with the Gamma-Gamma PDF can be applied to analyze the system performance in the cases with, e.g., the exponential distribution of the links. Also, while the presence of a battery improves the outage probability, its effect is negligible for moderate/large code rates (Fig. 1c).

\section{REFERENCES}

[1] S. Zhou, T. Chen, W. Chen, and Z. Niu, "Outage minimization for a fading wireless link with energy harvesting transmitter and receiver," IEEE J. Sel. Areas Commun., vol. 33, no. 3, pp. 496-511, March 2015.

[2] B. Makki, T. Svensson, and M. Zorzi, "Wireless energy and information transmission using feedback: Infinite and finite block-length analysis," IEEE Trans. Commun., vol. 64, no. 12, pp. 5304-5318, Dec. 2016.

[3] S. M. Aghajanzadeh and M. Uysal, "Information theoretic analysis of hybrid-ARQ protocols in coherent free-space optical systems," IEEE Trans. Commun., vol. 60, no. 5, pp. 1432-1442, May 2012.

[4] B. Makki, T. Svensson, T. Eriksson, and M.-S. Alouini, "On the performance of RF-FSO links with and without hybrid ARQ," IEEE Trans. Wireless Commun., vol. 15, no. 7, pp. 4928-4943, July 2016.

[5] Y. Tang and M. Brandt-Pearce, "Link allocation, routing and scheduling of FSO augmented RF wireless mesh networks," in Proc. IEEE ICC'2012, Ottawa, Canada, June 2012, pp. 3139-3143.

[6] Http://lasermotive.com/technology/power-beaming-overview/.

[7] X. Zhou, R. Zhang, and C. K. Ho, "Wireless information and power transfer: Architecture design and rate-energy tradeoff," IEEE Trans. Commun., vol. 61, no. 11, pp. 4754-4767, Nov. 2013.

[8] R. A. Horn and C. R. Johnson, Matrix Analysis. Cambridge University Press, 1985. 